\title{
The study design of UDRIVE: the naturalistic driving study across Europe for cars, trucks and scooters
}

\author{
Yvonne Barnard $^{1,2}$ • Fabian Utesch ${ }^{3}$ - Nicole van Nes ${ }^{4}$ Rob Eenink ${ }^{4}$. \\ Martin Baumann ${ }^{5}$
}

Received: 24 February 2015 / Accepted: 9 May 2016/Published online: 19 May 2016

(C) The Author(s) 2016. This article is published with open access at SpringerLink.com

\begin{abstract}
Purpose UDRIVE is the first large-scale European Naturalistic Driving Study on cars, trucks and powered two-wheelers. The acronym stands for "European naturalistic Driving and Riding for Infrastructure \& Vehicle safety and Environment". The purpose of the study is to gain a better understanding of what happens on the road in everyday traffic situations.

Methods The paper describes Naturalistic Driving Studies, a method which provides insight into the actual real-world behaviour of road users, unaffected by experimental conditions and related biases. Naturalistic driving can be defined as a study undertaken to provide insight into driver behaviour during everyday trips by recording details of the driver, the vehicle and the surroundings through unobtrusive data gathering equipment and without experimental control. Data collection will take place in six EU Member States.

Results Road User Behaviour will be studied with a focus on both safety and environment. The UDRIVE project follows the steps of the FESTA-V methodology, which was originally designed for Field Operational Tests.
\end{abstract}

This article is part of Topical collection on TRA 2014 human factors and safety

Yvonne Barnard

y.barnard@leeds.ac.uk

1 Institute for Transport Studies, University of Leeds, Leeds LS2 9JT, UK

2 ERTICO - ITS Europe, Brussels, Belgium

3 German Aerospace Center (DLR), Braunschweig, Germany

4 SWOV Institute for Road Safety Research, Leidschendam, The Netherlands

5 Institute of Psychology and Education, Department Human Factors, Ulm University, Ulm, Germany
Conclusions Defining research questions forms the basis of the study design and the specification of the recording equipment. Both will be described in this paper. Although the project has just started collecting data from drivers, we consider the process of designing the study as a major result which may help other initiatives to set up similar studies.

Keywords Naturalistic driving study $\cdot$ Road user behaviour . Road safety $\cdot$ Eco-driving

$\begin{array}{ll}\text { Nomenclature } \\ \text { DAS } & \text { Data acquisition system } \\ \text { FOT } & \text { Field operational test } \\ \text { ND } & \text { Naturalistic driving } \\ \text { NDS } & \text { Naturalistic driving study } \\ \text { SCE } & \text { Safety-critical event } \\ \text { UDRIVE } & \begin{array}{l}\text { eUropean naturalistic driving and riding } \\ \text { for infrastructure and vehicle safety and } \\ \text { environment }\end{array} \\ \text { VRU } & \text { Vulnerable road user }\end{array}$

\section{Introduction}

\subsection{Human behaviour in road transport}

Road transport is indispensable for the exchange of goods and persons within the European Union and between neighbouring countries. Unfortunately, road transport also has several major negative consequences, in particular those related to crashrelated fatalities and injuries, and to harmful emissions and the use of non-renewable energy.

Human behaviour is, directly or indirectly, an important determinant of these negative consequences. Consequently, 
an in-depth understanding of road user behaviour is needed to identify the main causes of, and the most promising approaches to mitigate the negative consequences. For example, how do road users behave in different conditions; how and when do normal traffic conditions or ordinary behaviour evolve into critical events or (near-)crashes; which factors affect driving style and related vehicle emissions, etc.?

Although there is a wealth of research on driver behaviour obtained in laboratory and simulator studies, there is a lack of understanding of what happens on the road in everyday traffic situations. Naturalistic Driving Studies (NDS) are a method to provide insight into the actual real-world behaviour of road users, unaffected by experimental conditions and related biases.

\subsection{Naturalistic driving}

A NDS can be defined as a study undertaken to provide insight into driver behaviour during everyday trips by observing in detail the driver, the vehicle and the surroundings through unobtrusive data gathering equipment and without experimental control [1]. Typically, in an NDS vehicles - passenger cars, trucks or scooters - are equipped with several small cameras and sensors. For several months to several years, these devices inconspicuously record vehicle manoeuvres (such as speed, acceleration/deceleration, direction), driver behaviour (such as eye, head and hand movements), and external conditions (such as road, traffic and weather characteristics). Thus, the ND approach allows us to observe and analyse the interrelationship between driver, vehicle, road and other traffic participants in ordinary situations, in conflict situations and, more rarely, in some actual crashes. This type of information is necessary for identifying new and promising measures, not only for reducing road transport casualties, but also for reducing the environmental impact of road transport.

Traditionally, road user behaviour research has been using driving simulators, instrumented vehicles, self-reports, analyses of crash statistics, and increasingly also in-depth crash investigation. These methods have greatly contributed to the understanding of road user behaviour. However, they also have several limitations. For example, results from driving simulator studies cannot always be easily transferred to real traffic situations, since both the traffic environment and the vehicle characteristics are only approximations of reality. This is especially true in the simpler and static-based simulators. In instrumented vehicle studies subjects drive in real traffic but in a special, highly equipped vehicle with, usually, an experimenter on-board. This makes subjects aware of the fact that they participate in an experiment which may affect their driving behaviour. The results of self-reports may be biased by socially desirable responses as well as by perceptual and memory limitations.
Compared to these traditional road safety research methods NDS has many important advantages. NDS also offers much wider perspectives in understanding normal traffic behaviour in normal everyday traffic situations. Participants are not involved in an experiment; there is no observer present, there are no experimental interventions or aims that participants can guess and act for. Furthermore, there is the possibility to observe conflicts, near-crashes or possibly even actual crashes without the potential biases of post-hoc reports. As such a NDS can contribute to clarifying the prevalence of, for example, fatigue and distraction among drivers/riders and the related crash risk; the interaction between road and traffic conditions and road user behaviour; to understanding the interaction between drivers/riders and vulnerable road users; to specifying the relationship between driving style and vehicle emissions and fuel consumption; and many other aspects of traffic participation that are difficult to study by means of traditional research.

\subsection{Experiences with NDS}

NDS is a fairly new research method that was developed in the late nineties of the previous century and that has been developed and refined continuously since. The method has become technically possible because of the tremendous developments in information and communication technologies, improvements in storage capacities, data-mining, image processing, low-cost camera technology, etc. in the last couple of decades.

The first major NDS was conducted in the USA by Dingus et al. [2]) who instrumented the cars of one hundred drivers who commuted on a regular basis in the Northern Virginia/ Washington D.C. metropolitan area. They gathered data over a 12-month period and during this time the vehicles were driven over $3,000,000 \mathrm{~km}$ with a total of $43,000 \mathrm{~h}$ of exposure involving 67 (mostly minor) crashes and 761 near-crashes. All vehicles were instrumented with a Data Acquisition System (DAS) engineered by Virginia Tech Transportation Institute (VTTI).

The large-scale follow-up study is part of the second Strategic Highway Research Program (SHRP2: www. shrp2nds.us; [3, 4]). Data acquisition is finished, and the SHRP2 database (https://insight.shrp2nds.us/) contains 2 petabytes of data from 5.4 million trips taken by 3147 volunteer drivers for between 4 and 24 months each - nearly 50 million miles of driving.

In Europe also there have been various, smaller-scale and more focused NDS that included ND methodologies. Several projects funded by the European Commission are:

- PROLOGUE: aimed to assess the feasibility and usefulness of a large-scale NDS in Europe and to formulate 
recommendations for such a large-scale study (www. prologue-eu.eu; [5]);

- INTERACTION: aimed at a better understanding of driver interactions with in-vehicle technologies (interactionfp7.eu).

- 2BeSafe: focusing on the behaviour and safety of powered two-wheelers (www.2besafe.eu; [6]).

- DaCoTA: assessing the usefulness of the ND method for gathering large-scale, representative information about safety performance indicators and exposure in the different EU Member States (www.dacota-project.eu; [7]).

- SeMiFOT: aimed at implementing and developing the Naturalistic Field Operational Test (N-FOT) method to understand crash causation and the effects of new safety systems [8].

- $\quad$ Large Field Operational Tests such as euroFOT (studying the use of Advanced Driver Support Systems, www. eurofot-ip.eu) and TeleFOT (studying the use of nomadic devices, www.telefot.eu) used NDS methods to investigate the behaviour of drivers while driving with new intelligent transport systems. While NDS tend to focus on crash-explanatory factors, Field Operational Tests generally focus on evaluation of systems or functions, however, the data collected in both types of studies can be used for many alternative purposes, such as analysis of Environment, Efficiency and Mobility impacts.

Also in other countries NDS have been performed or are ongoing, such as in Japan [9] and Australia (http://www.ands. unsw.edu.au/) [10].

In 2012 the European Commission decided to fund the first large scale European Naturalistic Driving Study: the UDRIVE project (www.udrive.eu). The acronym stands for "eUropean naturalistic Driving and Riding for Infrastructure and Vehicle safety and Environment". UDRIVE's objectives are two-fold: to identify well-founded and tailored measures to improve road safety, and to identify approaches for reducing harmful emissions and fuel consumption in order to make road traffic more sustainable.

In this paper we will first provide some more details on the project. Next we will describe the methodology the project followed to design this complex study. The process of defining research questions, and the results will be discussed. The research questions form the basis of the study design, and of the specification of the recording equipment. Both will be described. An outline will be given of how the study will be conducted at seven different European Operation sites. Finally, it will be explained how the data gathered in the project will be used, and what impact is to be expected. Although the project has just started collecting data from drivers, we consider the design of the study a major result which may help other initiatives to set up similar studies.

\section{The UDRIVE project}

The scientific and technical aims of UDRIVE are describing and quantifying road user behaviour in different European regions, in regular conditions and (near-)crashes, and providing a quantified estimate of the risk of particular safety-critical behaviours, focussing especially on the prevalence and effects of driver states, such as distraction and inattention, and the interactions between drivers/riders and high risk groups like pedestrians and cyclists. In addition, it aims at describing and quantifying road user behaviour in relation to emission levels and fuel consumption, focussing in particular on the effects of driving style, road and road network characteristics, and traffic conditions such as congestion, impaired visibility or adverse weather. The UDRIVE project does not only investigate the behaviour of drivers of passenger cars, but also that of truck drivers and scooter riders.

UDRIVE aims to provide recommendations for safety and sustainability measures related to regulation, enforcement, driver awareness, driver training, and road design. The UDRIVE results may lead to improved driver behaviour models and risk functions which can be used in traffic simulations. The project aims to making the traffic system safer and more sustainable, and identifying new approaches, measures and tools. Main results from the project will be the definition of measurable safety and environmental performance indicators for monitoring developments over time, the improvement of existing models of driver behaviour to be used for e.g. predicting effects of safety and environmental measures, and traffic flow simulations, and applications in commercial transport, including driver support systems and targeted training for safer and more fuel efficient driving. Finally UDRIVE will demonstrate how ND data can be used for commercial purposes, including safety and sustainability applications, behaviour based programmes and business models for future data collection.

The project is a 57 months large-scale integrating project, funded under the 7th EU Framework Programme. It started in 2012 and will end in 2017. Project partners are the SWOV (coordinator), BASt, CDV, CEESAR, CIDAUT, DLR, ERTICO, FIA, IBDIM, IFSTTAR, KFV, LAB, RENAULT, Loughborough University, Or Yarok, SAFER, TU Chemnitz, TNO, University of Leeds, VOLVO. More information may be found at the project website: http://www.udrive.eu/.

\section{Methodology}

The methodology to plan, design and conduct the NDS, and to analyse the results, is derived from the FESTA methodology for conducting Field Operational Tests (FOT) ([11]; FESTA, [12]). It was developed in the European FESTA project (Field opErational teSt supporT Action) by a consortium of a large 
number of partners, both industrial and academic. The methodology is currently maintained by the European Support Action FOT-Net (www.fot-net.eu), and has been revised several times. NDS are now included in this methodology. NDS and FOTs may be seen as different methods because the study design is different (participant selection, experimental conditions, vehicle sample etc.), and the research questions and hypotheses are different. In particular, the main difference relates to the degree of experimental control in the study. However, there is also substantial overlap, especially in the structured approach to develop the study, and the tools and analysis methods used. Between the FOT and the NDS lies the Naturalistic FOT. This last one uses observation in a natural setting, typically to evaluate the relationship between driver-, vehicle-, or environment factors and crash risk, driving behaviour, and the effectiveness of countermeasures [8].

In Fig. 1 the FESTA methodology is summarised, and the steps relevant for NDS encircled. The methodology is described in detail in the FESTA handbook (FESTA, [12]). The methodology consists of a process which systematically details the steps to be taken to set up the test (the left-hand side of the V), the actual data acquisition (the bottom of the $\mathrm{V}$ ), and the analysis of the data and evaluation and interpretation of the results (the right-hand side of the V). The first part of the methodology to define the study follows a systematic research oriented approach. For Field Operational Tests the process starts with defining functions and systems to be investigated (for example, a forward collision warning), the use cases have to be defined, specific events in which a system is expected to behave according to the specified function, for example, car following. For Naturalistic Driving studies, this is not relevant, so the methodology starts with the next step: the definition of research questions and hypotheses. Defining research questions is a major step; below we will describe how this step was taken in UDRIVE. The study is then designed in detail and performance indicators are selected. Performance indicators are quantitative or qualitative indicators, monitored at regular or irregular intervals, and can be compared to one or more criteria (for example acceleration). The next step determines which specific measures and sensors to use. When the whole test is defined, the actual data acquisition can take place. Data will be stored in a database and analysed. Analysis leads to evaluation of whether the research questions have been answered. In a FOT the next steps concern evaluating the functioning of the systems and answering the question of what the impact would be if the system were fully deployed in a large proportion of vehicles. Naturalistic driving studies have a different aim, and therefore these steps are not relevant. However, NDS also have scaling up activities, such as the aggregation of results, and the analysis of the implications of these results. For example, one can use findings to identify new and more efficient measures and tools to improve safety and sustainability of road transport. Another impact area is to demonstrate how naturalistic driving can be used in the industrial development of safety and sustainability functions and services.

From the very beginning and throughout the project ethical and legal questions should be addressed, such as the privacy of the participants, responsibilities and data ownership. In NDS participants are observed for a long period by data acquisition systems including video cameras, and data may also be gathered from the environment and other road users. It should be clear that these are not trivial issues. Moreover, in a European context a NDS project has to deal with different regulations and legislation in the member states.
Fig. 1 The FESTA methodology, with the steps relevant for NDS encircled

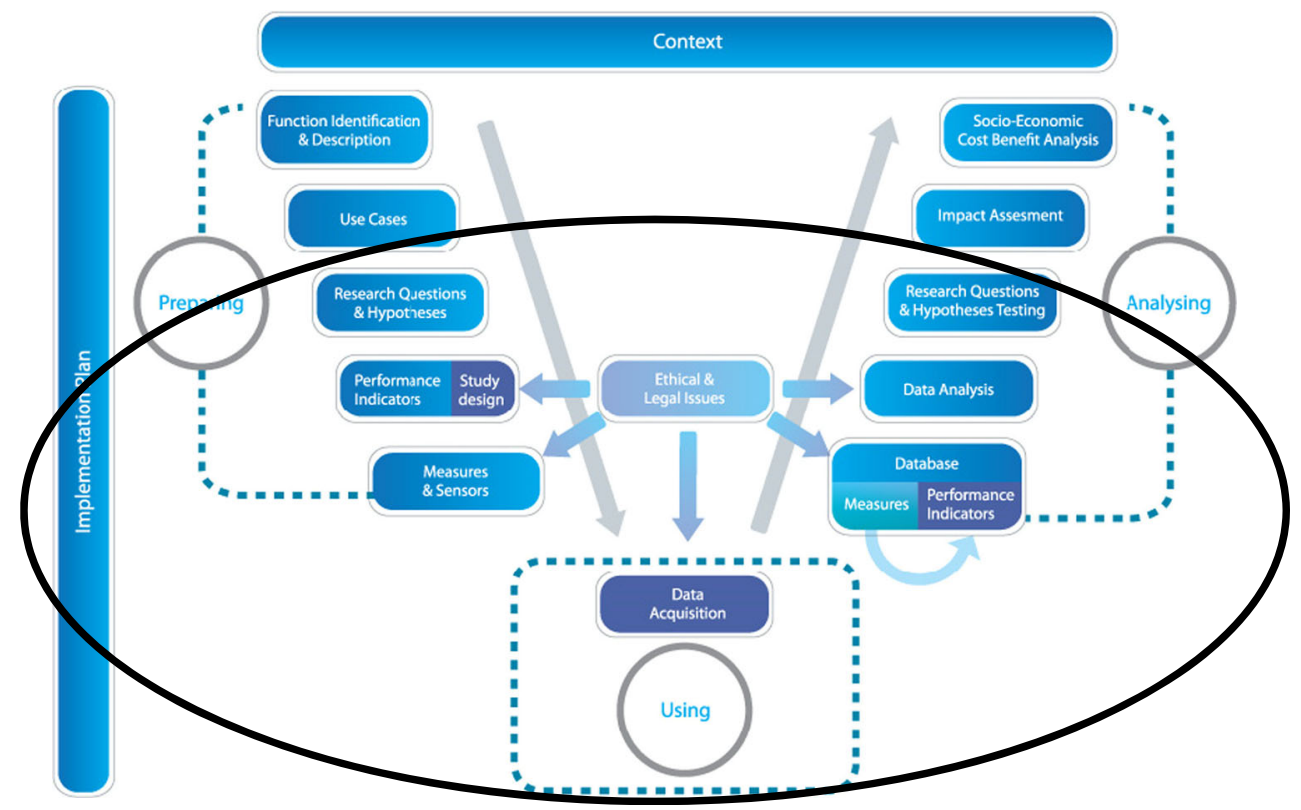


In the FESTA methodology, two processes are depicted outside the V. The first is the horizontal Context bar. This includes (see also [13]) the identification of stakeholders, the selection of topics, the dissemination activities, and last but not least the identification of constraints, such as available technologies and budgets. As a NDS is a long, complex and costly project, this part of the methodology is crucial for the success of the project. The FOTIP is depicted in the vertical bar: this is the FOT Implementation Plan, the FESTA handbook provides a wealth of practical details and a checklist to ensure that all necessary steps and actions are taken care of.

The UDRIVE project follows the FESTA methodology and has divided its subprojects (SP) according to (clusters of) FESTA V steps, see Fig. 2. The implementation plan is included in SP1, 2, 3, 4 and 5. Although we mentioned before that impact analysis is not necessarily part of a NDS, UDRIVE has a special SP devoted to this, focussing on the impact of the SP4 results, by generating guidelines and recommendations in some specific areas such as safety and sustainability.

Additional to the classical FESTA V, and indicated by the arrows between SP1 and SP4 and SP5 in Fig. 2, a close link between these SPs was established explicitly from the beginning in order to ensure that the analysis goals lying behind the research questions are clear to the analysts when analysing the data later on, and that known analysis constraints of NDS data are considered already during the development of the research questions.

\section{Research questions and required data}

In UDRIVE, research questions were developed based on a review of existing research activities relevant for traffic safety and eco-driving. A subsample of these questions were selected for analysis in UDRIVE based on the resources, technical possibilities and data collected. This first comprehensive list of research questions enabled us to derive technical requirements for the data acquisition system, and thus laid the foundation to enable researchers to address further questions even after the end of the project. This list contains 32 questions distributed across the five thematic areas: Crash causation and risk, Everyday driving, Distraction and inattention, Vulnerable road users and Eco-driving. It is outside the scope of this paper to list all these research questions. The same is true for the list of over 100 measures. Instead a short description of each research area including examples and important related measures will be given below.

Crash causation and risk This topic focuses on identifying factors that can cause or contribute to a crash and increase the crash risk. Many potential relevant factors are recorded and their presence in safety-critical events (SCEs) will be compared to baseline events with odds ratios. Extreme peaks in the sensor data, such as unusual hard braking events, quick steering manoeuvres or high longitudinal or lateral accelerations, will be used to identify SCEs. Baseline events will be drawn from data that do not include such events. To accommodate the different needs of the research questions these baseline events will be selected randomly as well as stratified according to predefined criteria in order to have comparable situations with regard to the identified SCEs from all available trips. Risks will be calculated for engaging in secondary tasks, looking away from the road, being drowsy and driving riskily. Additionally this task will look into chains of contributing factors to reveal patterns leading to specific critical events. The analysis will mostly rely on video data. Sensor data will be used to identify relevant episodes for video annotation.
Fig. 2 The UDRIVE work programme

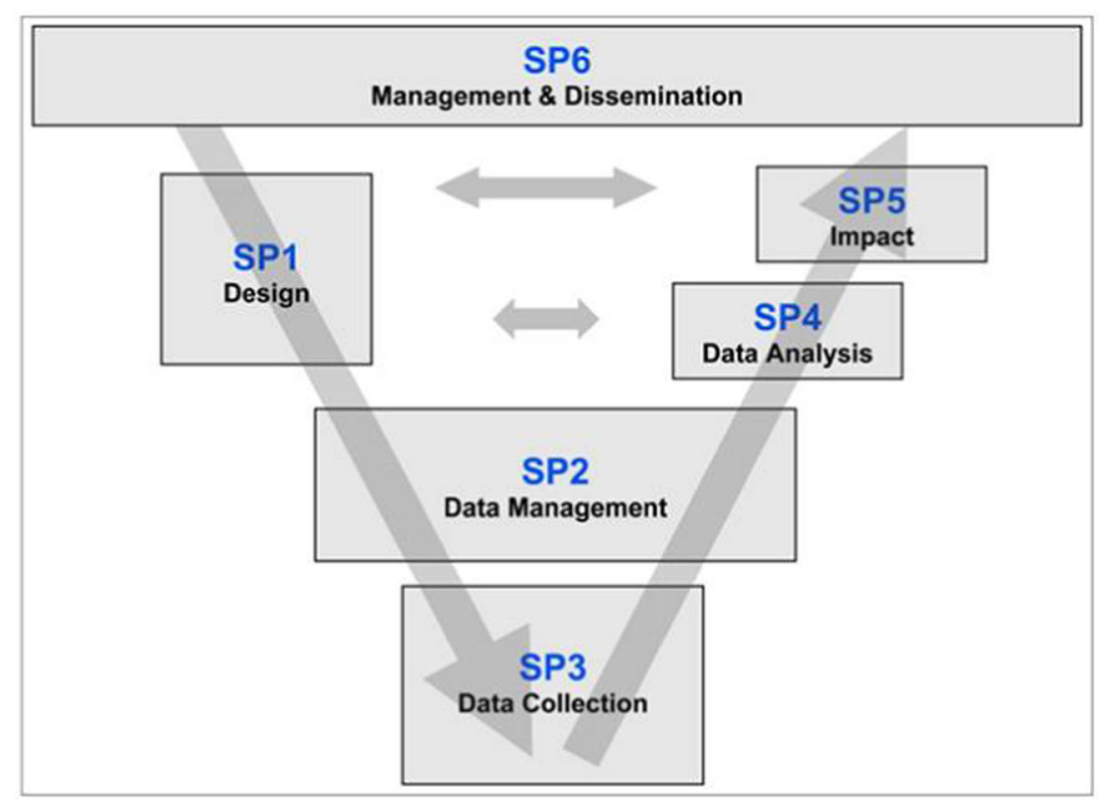


Video analysis will then be used to verify whether a candidate event is indeed a critical event. By means of video annotation relevant factors will be extracted that cannot be directly reliably measured yet, such as secondary task performance. Safety-critical and baseline events will be the main data source for answering the research questions. For the selection of relevant events, the activity of the brake pedal, steering wheel, accelerometers as well as information (i.e. distance measures, and warnings) from a smart camera will be used.

Three examples:

- What are the risks of different driver behaviours? Risks of several secondary tasks will be evaluated focusing on visual-manual distracting factors such as operating a phone, tablet, or other devices or objects while driving.

- Is there a difference in driving related risks under different conditions? In UDRIVE data are collected across several European countries. Therefore the risks calculated above will be compared between countries and modalities such as road and vehicle type.

- How can contributing-factor chain schemas be applied to naturalistic road user data? The aim is to further develop a qualitative coding scheme of factors contributing to a safety-critical event. A method will be developed helping to describe possible causation chains and weighing individual paths that are observed in the naturalistic driving data. Thus important reaction chains may be revealed.

Everyday driving The aim is to gain more insight in what happens on the road on a day to day basis trying to understand who will engage in risky driving behaviour when and where. To achieve this, this research area includes questionnaire data that is acquired in the beginning of the study. Driver characteristics such as age, gender, driving experience, sensation seeking [14] and locus of control [15] are assessed. Additionally drivers fill in the Driver Behaviour Questionnaire (DBQ; Parker et al. 1995), the Driver Attitude Questionnaire (DAQ; [16]) and the Driving Style Questionnaire (DSQ, [17]). This battery of driver characteristics will be compared to the actual driving behaviour (i.e. risk taking and potential conflicts). Relevant variables of the vehicle include video data identifying situational variables inside the vehicle, for example, whether and how many passengers were present and whether a seatbelt was worn. Additionally using video data, temporal factors (day/night, rush hours) can be identified. The positioning data will be used to identify the environment road type (single carriageway, motorway), road geometry (intersection, link, bend, junction), locality (urban, rural). Drivers' characteristics will be compared with risk managing behaviours such as maintaining a greater safety distance. Data acquired with vehicle- and built-in sensors together with a smart camera will be utilized to derive driving patterns. They allow measuring speeding, time headway and lateral g-force to identify risky manoeuvres.
Three examples:

- To what extent are driver factors associated with risky behaviour? The aim is to gain more insight in who engages in risky behaviour, and the relation between driver characteristics and speed choice, curve negotiation and car following behaviour.

- To what extent are environmental factors associated with risky behaviour? More information will be gathered on who drives on what types of road, and what environmental and infrastructural characteristics influence driver behaviour.

- To what extent are seatbelts used? Here driver characteristics, environmental factors and trip characteristics (short/ long, presence of passengers) will be compared to actual seatbelt use.

Distraction and inattention From existing research we know that especially secondary tasks diminish drivers' ability to control the vehicle safely. Thus a research area was dedicated to looking into attention selection mechanisms and the willingness of drivers to engage in secondary tasks. This area aims to understand why drivers are distracted and how they adapt when distracted. Video data will be used assessing the situation in front of the driver as well as identifying safety-critical events. A close view of the driver face is needed to see the gaze direction and a view inside the vehicle to identify whether and which secondary tasks are performed. A smart camera measures the distance to potential crash objects and pedal positions, vehicle speed, longitudinal and lateral accelerometers will be used to identify driver reactions such as avoidance manoeuvres. Positioning data will be used to determine road type and other environmental variables.

Three examples:

- Which perceptual cues reliably capture attention and trigger avoidance manoeuvres in SCEs? Sudden onsets or optical expansion of objects capture visual attention (e.g. [18]). The aim is to better understand how these factors work in a driving scenario. Externally triggered glances will be identified and it will be determined whether they were beneficial or detrimental to the driving task.

- Why do the reactive attention capture mechanisms, identified above, sometimes fail and lead to crashes? As a companion question to the afore-mentioned one, the focus is on different forms of inattention including fatigue, sleepiness and cognitive load.

- What factors determine how drivers proactively allocate their attention in anticipation of how a driving situation will unfold, and why do these proactive selection mechanisms sometimes fail? This question is targeted at finding 
out why drivers did not have their eyes on the road when a lead vehicle braked.

Vulnerable road users A lot of research focuses on drivers of cars or trucks. Not much is known about road users who are not protected by a chassis. Pedestrians, cyclists and motorcyclists belong to this group and have a high risk of serious injury when involved in a crash (ETSC, [19]). Research questions within this research area aim to describe SCEs and to find ways to avoid critical incidents related to these road users. Such incidents can be analysed from two perspectives within the UDRIVE project: from the driver perspective but also from the perspective of one group of vulnerable road users, namely scooter riders as the UDRIVE driver fleet features scooters offering the unique perspective of vulnerable road users (VRUs) themselves. The analysis will rely on a smart camera system with which the cars and trucks are equipped, which is able to identify and track different road users in front of the vehicle. Additionally, video will be crucial for identifying what was happening in front of the vehicle and where the driver was looking. In the vehicles a cabin camera can reveal drivers' secondary task engagement. The data will be correlated with driver and road characteristics, weather and light conditions. These research questions require a detailed description of road users surrounding the vehicle, such as pedestrians (conspicuity, distance, number, estimated age and gender). Videos will be annotated in order to derive the information.

Three examples:

- What characterizes SCEs involving motorised traffic and cyclists at intersections? The causes and build-up of SCEs at intersections will be investigated.

- How do car drivers behave at intersections in urban areas where they might encounter cyclists? Which 'external' factors (e.g. intersection design) modify those behaviours? This research question focuses on the driver's speed and yielding behaviour when encountering VRUs while turning.

- How do drivers and riders differ on speed choice? Specific research questions focus on riders of powered two-wheelers. UDRIVE will compare the riding to driving behaviour to better understand the needs of and interaction between both. This research question focuses on speed while another one is looking at behaviour.

Eco-driving A concern becoming more prominent every year is the protection of our environment. Emissions contribute to global warming and pollute the air. Especially in large cities health risks caused by traffic pollution are of growing concern. The ability to reduce fuel consumption and $\mathrm{CO} 2$ emissions by driving style can be called eco-driving. Consequently this research area focuses on understanding when, how and why
Table 1 UDRIVE fleets type and size per country

\begin{tabular}{lll}
\hline Type of vehicle & Country & $\begin{array}{l}\text { Fleet size (number } \\
\text { of DAS) }\end{array}$ \\
\hline Car & France & 30 \\
& Germany & 20 \\
& Netherlands & 10 \\
& Poland & 30 \\
& UK & 30 \\
Powered Two-wheelers & Spain & 40 \\
Truck & Netherlands & 40 \\
& & 200 \\
\hline
\end{tabular}

drivers drive with low fuel consumption. The relevant data for eco-driving consists of all continuous measurements. Driving speed, longitudinal and lateral acceleration as well as road inclination from positioning data are crucial for the analysis. Additional important measurements are headway, gear shifting and pedal use, current speed limit, the presence of traffic lights and engine speed.

Three examples:

- Does the vehicle power-to-mass ratio affect the driving style?

- How much do drivers deviate from the speed limit in free flow situations, and why?

- When do drivers brake, and is it necessary to brake in each instance?

The type of data required to answer the research questions of the different research areas was defined. For each thematic area the scenarios, the candidate SCEs, the baseline, and further characteristics of the dataset were identified. In terms of scenarios, the context in which data needs to be acquired was described. This includes the tasks performed by the road user (e.g. car following, negotiating bends; overtaking; free flow), and the characteristics of the environment (road type, traffic conditions, time of day). With regard to the candidate SCEs a description of critical situations that are relevant to be

Table 2 Specification of DAS features per vehicle type

\begin{tabular}{lll}
\hline Car & Truck & Scooter \\
\hline 7 cameras & 8 cameras & 5 cameras \\
IMU sensors & IMU sensors & IMU sensors \\
GPS & GPS & GPS \\
Mobil Eye smart camera & Mobil Eye smart camera & $\mathrm{X}$ \\
CAN data & CAN data & $\mathrm{X}$ \\
Sound level & Sound level & $\mathrm{X}$ \\
\hline
\end{tabular}



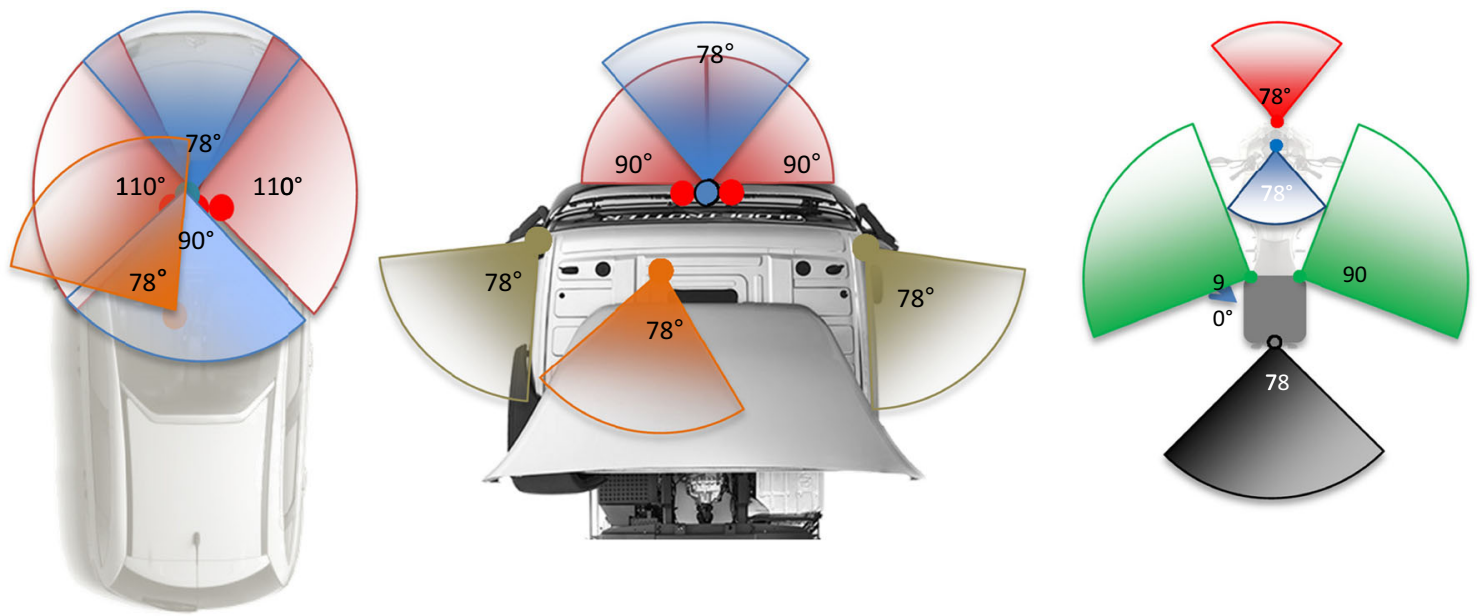

Fig. 3 The camera views collected for trucks, cars and scooters

analysed was given, and of how these can be selected (within boundaries). Furthermore, the baseline scenarios to be used as a comparable reference, and the characteristics of the dataset were clarified, in order to better understand how the research questions can be addressed.

\section{Participants and vehicles}

The research questions and the detailed data needs, as described in the section above, formed the basis for designing a study plan. The data collection involves three types of vehicles across six countries, see Table 1 for a specification of the vehicle type and number per country, and they will drive for 18 months. The aim is to recruit as much as possible vehicles with multiple drivers, in order to recruit more participants. The Dutch Operational Site will run three waves of participants for about 6 months per wave, resulting in 30 participants. The number of vehicles involved is limited due to the resources available but allows for sufficient data to answer research questions,
Requirements for the participants were defined with regards to experience, age and gender. These factors are seen as the most relevant considering the research questions. With respect to vehicles, the study focusses on three types of passenger cars (Renault Megane, Renault Clio 3, Renault Clio 4), one type of powered two-wheelers (Piaggio scooter), and two types of trucks (Volvo FM and Volvo FL).

\section{Data acquisition}

Research questions and study plan fed into the specifications for the Data Acquisition System (DAS). It was strongly desired to have one common DAS for the three different vehicle types. This common DAS was tailored to the needs of the project and to fit to the three different vehicle types (e. g. rugged sensors or cameras for scooter or similar). The data logger is the same for all vehicle types. The number of cameras and other sensors is adapted to the vehicle type. This is specified in Table 2 .
Fig. 4 Impression of UDRIVE video data in draft version of analysis tool

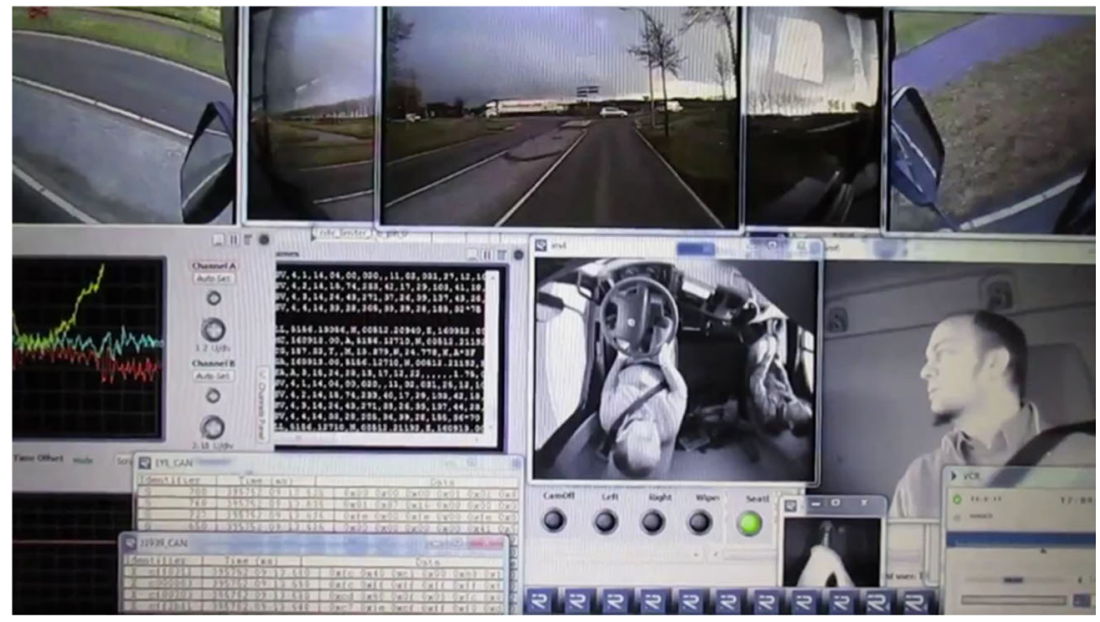


The high number of camera views and the quality of the camera views are unique for a large scale Naturalistic driving study. Figure 3 provides an overview of the camera views of the different vehicles. They provide a good look around the vehicle and inside the vehicle for the trucks and cars and a view of the driver for the scooters.

The data is being collated at seven Operation Sites (OS). This data is pre-processed at three Local Data Centres (LDCs). During the pre-processing the data is enriched with e.g. map data (i.e., the addition of road and infrastructure attributes from digital maps). The pre-processed data is to be stored at a central data centre (CDC). The partners have remote access to the data at the CDC. All analysis and annotation are done directly on the central data set at the CDC through the remote access. To facilitate processing, annotation and analysis of the data, a common software toolset is developed within the project. For all trips the driver ID will be confirmed based on the first video frames. In this step the trip is matched to a driver, as more than one driver per vehicle can participate in the study. If the driver in question is not a participant, but maybe someone that drives this vehicle incidentally, the trip is deleted in order to protect the privacy of non-participants. Figure 4 gives an impression of the video data collected. This is an example of the truck data in a draft version of the analysis tool.

The data collected within the project will be available for further research after the project for partners and also for third parties, with certain limitations to adhere to the privacy of the participants as agreed in the informed consent forms.

\section{Expected results and conclusions}

On the Operation Sites participants are recruited and their vehicles are instrumented with the DAS. They will drive and ride with this equipment for a year and a half, and their data will be gathered. The data will be analysed and the results will provide answers to the research questions. Not all results will provide new insight, we expect to find information on driver behaviour already known from observations by human observers and from experimental and simulator studies; our study will be able to validate these findings. However, we also expect to find new insight and more precise insight on what is actually happening on European roads and how drivers and riders behave in normal situations, in critical and dangerous situations, and in interaction with other road-users. This knowledge is needed by stakeholders who make decisions about transport policies, development of new intelligent transport systems, and for training of drivers and riders. UDRIVE aims to provide recommendations for safety and sustainability measures related to regulation, enforcement, driver awareness, driver training, and road design. The UDRIVE results may lead to improved driver behaviour models and risk functions which can be used for traffic simulations. Finally UDRIVE will demonstrate how ND data can be used for commercial purposes, including safety and sustainability applications, behaviour based programmes, and business models for future data collection.

After the end of the project the UDRIVE project will offer access (within the bounds of legal and ethical restrictions) to the collected data so that it can be consulted and used for subsequent analyses by road safety and environmental experts from all over the world. This will enable the exploitation of the data beyond the scope of the UDRIVE project.

Acknowledgments UDRIVE is co-funded by the European Commission, DG Research and Innovation, in the 7th Framework Programme, GA No. 314050. The authors would like to thank all UDRIVE project partners for their contributions to the study.

Open Access This article is distributed under the terms of the Creative Commons Attribution 4.0 International License (http:// creativecommons.org/licenses/by/4.0/), which permits unrestricted use, distribution, and reproduction in any medium, provided you give appropriate credit to the original author(s) and the source, provide a link to the Creative Commons license, and indicate if changes were made.

\section{References}

1. Van Schagen I, Welsh R, Backer-Grøndahl A, Hoedemaeker M, Lotan T, Morris A, Sagberg F, Winkelbauer M (2011) Towards a large-scale European Naturalistic Driving study: main findings of PROLOGUE. PROLOGUE Deliverable D4.2. Leidschendam, The Netherlands, SWOV Institute for Road Safety Research

2. Dingus TA, Klauer SG, Neale VL, Petersen A, Lee SE, Sudweeks J, Perez MA, Hankey J, Ramsey D, Gupta S, Bucher C, Doerzaph ZR, Jermeland J, Knipling RR (2006) The 100-car naturalistic driving study, phase II: results of the 100-car field experiment. NHTSA report DOT HS 809 593. NHTSA, Washington, US

3. Antin JF (2011) Design of the in-vehicle driving behavior and crash risk study: in support of the SHRP 2 naturalistic driving study. Washington D.C, Transportation Research Board

4. Boyle LN, Lee JD, Neyens DM, McGehee DV, Hallmark S, Ward NJ (2009) SHRP2 S02 integration of analysis methods and development of analysis plan. Phase 1 report. University of Iowa, Iowa City, IA

5. Sagberg F, Eenink R, Hoedemaeker M, Lotan T, Van Nes N, Smokers R, Winkelbauer M (2011) Recommendations for a largescale European naturalistic driving observation study. PROLOGUE deliverable D4.1. Oslo, Norway, TØI Institute of Transport Economics

6. Laporte S, Espié S (2012) 2-BE-SAFE Project final report. Noisy le grand. France, IFSTTAR

7. Thomas P, Muhlrad N, Hill J, Yannis G, Dupont E, Martensen H, Hermitte T, Bos N (2013) Final Project Report, Deliverable0.1 of the EC FP7 project DaCoTA. Transport Safety Research Centre, Loughborough University, Loughborough, UK

8. Victor TW, Bärgman J, Hjälmdahl M, Kircher K, Svanberg E, Hurtig S, Gellerman H, Moeschlin F (2010) Sweden-Michigan naturalistic field operational test (SeMiFOT) phase 1: Final report." SAFER Report 2. Gothenburg, Sweden, SAFER

9. Uchidaa N, Kawakoshib M, Tagawab T, Mochidac T (2010) An investigation of factors contributing to major crash types 
in Japan based on naturalistic driving data. IATSS Res 34(1):22-30

10. Regan, M. A., Williamson, A., Grzebieta, R., Charltonb, J., Lenneb, M., Watsonc, B., Haworth, N. Rakotonirainy, A., Woolley, J., Anderson, R., Senserrick, T., \& Young, K. The Australian 400-car Naturalistic Driving Study: Innovation in road safety research and policy. In: Proceedings of the Australasian Road Safety Research, Policing and Education Conference. Proceedings of the 2013 Australasian Road Safety Research, Policing \& Education Conference 28th - 30th August, Brisbane, Queensland. Australasian Council of Road Safety (2013).

11. Barnard Y, Carsten O (2010) Field Operational Tests: challenges and methods. In: Krems J, Petzoldt T, Henning M (eds) Proceedings of European Conference on Human Centred Design for Intelligent Transport Systems, Eds edn. HUMANIST publications, Lyon, pp. 323-332

12. FESTA Consortium FESTA Handbook Version 5, revised by FOTNet. Available from: http://fot-net.eu/Documents/festa-handbookversion-5-2014 (2014).
13. Sagberg F, Backer-Grøndahl A (2010) A catalogue of applications and research topics for future naturalistic driving studies. PROLOGUE deliverable D1.3. Oslo, Norway, TØI Institute of Transport Economics

14. Arnett J (1994) Sensation seeking: a new conceptualization and a new scale. Personal Individ Differ 16(2):289-296

15. Özkan T, Lajunen T (2005) Multidimensional traffic locus of control scale (T-LOC): factor structure and relationship to risky driving. Personal Individ Differ 38(3):533-545

16. Parker D, Stradling SG, Manstead A (1996) Modifying beliefs and attitudes to exceeding the speed limit: an intervention study based on the theory of planned behaviour. J Appl Soc Psychol 26:1-19

17. French DJ, West RJ, Elander J, Wilding JM (1993) Decisionmaking style, driving style, and self-reported involvement in road traffic accidents. Ergon 36:627-644

18. Franconeri SL, Simons DJ (2003) Moving and looming stimuli capture attention. Percept Psychophys 65:999-1010

19. ETSC Bikepal ranking. Brussels, Belgium: European Traffic Safety Council (ETSC) (2012). 